\title{
UNIVERSITYOF
}

FORWARD

THINKING

WESTMINSTER用

WestminsterResearch

http://www.westminster.ac.uk/westminsterresearch

\section{Design and Simulation of a 3rd-order Discrete-Time Time- Interleaved Delta-Sigma Modulator with Shared Integrators between Two Paths}

Talebzadeh, J. and Kale, I.

This is a copy of the author's accepted version of a paper subsequently to be published in the proceedings of the 25th Iranian Conference on Electrical Engineering (ICEE2017), Tehran, Iran 02 to 04 May 2017, IEEE.

It is available online at:

https://dx.doi.org/10.1109//ranianCEE.2017.7985431

(C) 2017 IEEE . Personal use of this material is permitted. Permission from IEEE must be obtained for all other uses, in any current or future media, including reprinting/republishing this material for advertising or promotional purposes, creating new collective works, for resale or redistribution to servers or lists, or reuse of any copyrighted component of this work in other works.

The WestminsterResearch online digital archive at the University of Westminster aims to make the research output of the University available to a wider audience. Copyright and Moral Rights remain with the authors and/or copyright owners.

Whilst further distribution of specific materials from within this archive is forbidden, you may freely distribute the URL of WestminsterResearch: ((http://westminsterresearch.wmin.ac.uk/).

In case of abuse or copyright appearing without permission e-mail repository@westminster.ac.uk 


\title{
Design and Simulation of a $3^{\text {rd }}$-order Discrete-Time Time-Interleaved Delta-Sigma Modulator with Shared Integrators between Two Paths
}

\author{
Jafar Talebzadeh and Izzet Kale \\ Applied DSP \& VLSI Research Group \\ Department of Engineering \\ University of Westminster London, W1W 6UW, UK \\ Emails: Jtalebzadeh@gmail.com \& kalei@westminster.ac.uk
}

\begin{abstract}
, this paper presents the design and simulation of a $3^{\text {rd }}$-order two-path Discrete-Time Time-Interleaved (DTTI) $\Delta \Sigma$ modulator. By exploiting the concept of the time-interleaving techniques and time domain equations, a conventional $3^{\text {rd }}$-order Discrete-Time (DT) $\Delta \Sigma$ modulator is converted to a corresponding $3^{\text {rd }}$-order two-path DTTI counterpart. For the sake of saving power and silicon area, the integrators between the two paths of the DTTI $\Delta \Sigma$ modulator are shared. Using one set of integrators makes the DTTI $\Delta \Sigma$ modulator robust to path mismatch effects compared to the typical DTTI $\Delta \Sigma$ modulator which has individual integrators in all paths. A problem arises out of sharing integrators between paths which we call the delayless feedback problem. A solution for this problem is proposed in this paper and for an OverSampling Ratio (OSR) of 16 and a clock frequency of $320 \mathrm{MHz}$, a maximum SNR of $76.5 \mathrm{~dB}$ is obtained.
\end{abstract}

Keywords: Discrete-Time, Time-Interleaved, $\Delta \Sigma$ modulator, Signal-to-Noise Ratio, OverSampling Ratio.

\section{INTRODUCTION}

The $\Delta \Sigma$ modulators can achieve a very high resolution analog-to-digital conversion for low bandwidth applications by using oversampling and noise shaping techniques[1]. Recently, wideband applications require the ADCs with higher signal bandwidth. In order to increase the signal bandwidth of the modulator, a variety of methods can be used such as: using higher sampling frequency, increasing the order of the modulator and the number of quantizer bits.However, each of them has a price and is limited by technology [2].

One efficient appoach to increase the signal bandwidth is to use the time-interleaving technique [3] which increases the effective sampling frequency by paralleling more channels. This method is discussed in the next section.

\section{TIME-INTERLEAVED (TI) $\Delta \Sigma$ MODULATORS}

The well known procedure for the design of a $\Delta \Sigma$ modulator is based on choosing: the order and architecture of the $\Delta \Sigma$ modulator, the OSR and the number of bits for the quantizer. The time-interleaving technique can be successfully applied to $\Delta \Sigma$ modulators. However, the performance of the whole modulator is degraded due to channel mismatches. By paralleling $\mathrm{M}$ interconnected modulators that are working concurrently, the effective sampling rate and the OSR becomes $M$ times the clock rate and the OSR of each modulator respectively [4],[5]. It should be noted that the required resolution can be obtained without increasing the order of the modulator or the number of bits for the quantizer and also without utilizing a state of the art technology.

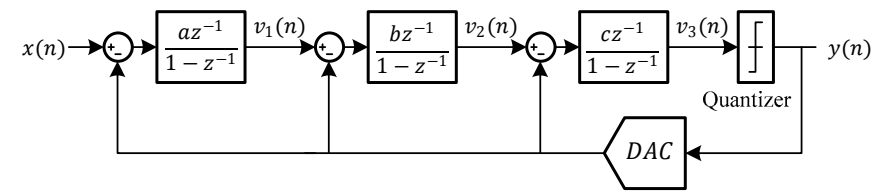

Figure 1: A $3^{\text {rd }}$-order conventional single-loop DT $\Delta \Sigma$ modulator.

\section{A. DERIVATION OF THE TI $\Delta \Sigma$ MODULATOR}

The $3^{\text {rd }}$-order DTTI $\Delta \Sigma$ modulator is derived directly from the time domain node equations of its conventional DT $\Delta \Sigma$ modulator as shown in Figure 1. It is assumed that the DAC in the feedback loop is ideal $\left(H_{D A C}(z)=1\right)$. The time domain equations of the modulator are written for two consecutive time slots as $(2 n)^{\text {th }}$ and $(2 n+1)^{\text {th }}$ as follows [6]:

$v_{1}(2 n)=a x(2 n-1)-a y(2 n-1)+v_{1}(2 n-1)$
$v_{2}(2 n)=b v_{1}(2 n-1)-b y(2 n-1)+v_{2}(2 n-1)$
$v_{3}(2 n)=c v_{2}(2 n-1)-c y(2 n-1)+v_{3}(2 n-1)$
$y(2 n)=Q\left[v_{3}(2 n)\right]$

and

$v_{1}(2 n+1)=a x(2 n)-a y(2 n)+v_{1}(2 n)$
$v_{2}(2 n+1)=b v_{1}(2 n)-b y(2 n)+v_{2}(2 n)$
$v_{3}(2 n+1)=c v_{2}(2 n)-c y(2 n)+v_{3}(2 n)$
$y(2 n+1)=Q\left[v_{3}(2 n+1)\right]$

where $Q[$.$] represents the quantization function. The input$ $x(n)$ is distributed between two channels through an input multiplexer which operates at twice the clock frequency of each channel. The input $x(n)$ is relabelled as follows:

$x_{1}(n)=x(2 n), \quad x_{2}(n)=x(2 n-1)$ 


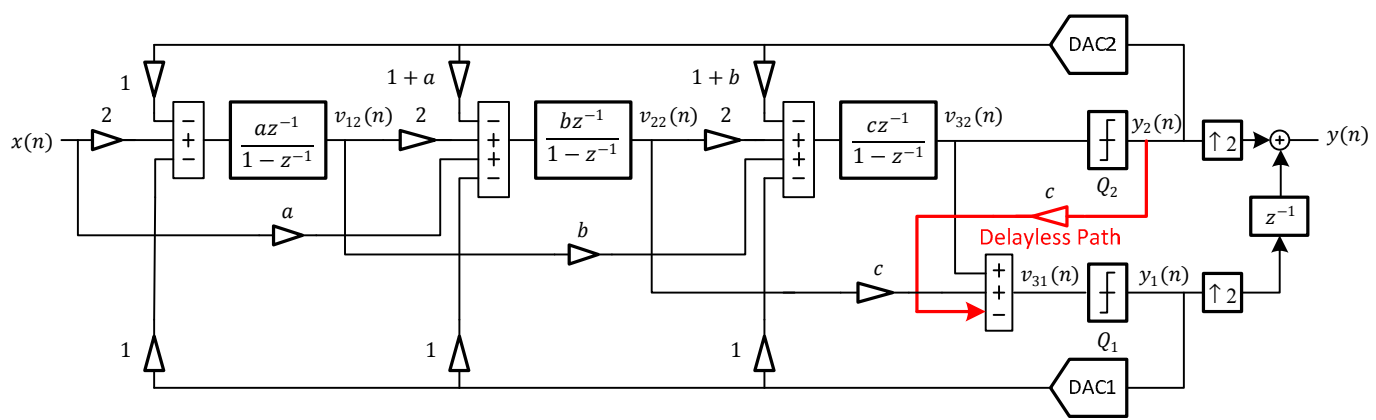

Figure 2: A $3^{\text {rd }}$-order DTTI $\Delta \Sigma$ modulator with shared integrators and delayless feedback path.

Similarly, the other nodes of the modulator are relabelled:

$$
\begin{array}{ll}
v_{11}(n)=v_{1}(2 n), & v_{12}(n)=v_{1}(2 n-1) \\
v_{21}(n)=v_{2}(2 n), & v_{22}(n)=v_{2}(2 n-1) \\
v_{31}(n)=v_{3}(2 n), & v_{32}(n)=v_{3}(2 n-1) \\
y_{1}(n)=y(2 n), & y_{2}(n)=y(2 n-1)
\end{array}
$$

By sharing only one set of integrators, the input demultiplexer is removed and the input $x(n)$ is shared between channels. Hence equation (3) results in (5) as follows:

$x_{1}(n)=x_{2}(n)=x(n)$

Equation sets (6) and (7) are derived by substituting equation set (4) and equation (5) into equation sets (1) and (2) respectively as follows:

$$
\begin{aligned}
& v_{11}(n)=a x(n) \quad-a y_{2}(n)+v_{12}(n) \\
& v_{21}(n)=b v_{12}(n)-b y_{2}(n)+v_{22}(n) \\
& v_{31}(n)=c v_{22}(n)-c y_{2}(n)+v_{32}(n) \\
& y_{1}(n)=Q\left[v_{31}(n)\right]=Q\left[c v_{22}(n)-c y_{2}(n)+v_{32}(n)\right]
\end{aligned}
$$

and

$$
\begin{aligned}
& v_{12}(n+1)=a x(n)-a y_{1}(n)+v_{11}(n) \\
& v_{22}(n+1)=b v_{11}(n)-b y_{1}(n)+v_{21}(n) \\
& v_{32}(n+1)=c v_{21}(n)-c y_{1}(n)+v_{31}(n) \\
& y_{2}(n+1)=Q\left[v_{32}(n+1)\right]
\end{aligned}
$$

Equation set (7) can be rewritten as equation set (8):

$$
\begin{aligned}
& v_{12}(n)=a x(n-1)-a y_{1}(n-1)+v_{11}(n-1) \\
& v_{22}(n)=b v_{11}(n-1)-b y_{1}(n-1)+v_{21}(n-1) \\
& v_{32}(n)=c v_{21}(n-1)-c y_{1}(n-1)+v_{31}(n-1) \\
& y_{2}(n)=Q\left[v_{32}(n)\right]
\end{aligned}
$$

Equation set (9) is derived by further substituting equation set (6) into equation set (8).

$$
\begin{aligned}
& v_{12}(n)=2 a x(n-1)-a y_{1}(n-1)-a y_{2}(n-1)+ \\
& v_{12}(n-1) \\
& v_{22}(n)=a b x(n-1)+2 b v_{12}(n-1)-b y_{1}(n-1)- \\
& b(1+a) y_{2}(n-1)+v_{22}(n-1) \\
& v_{32}(n)=b c v_{12}(n-1)+2 c v_{22}(n-1)-c y_{1}(n-1)-
\end{aligned}
$$

$c(1+b) y_{2}(n-1)+v_{32}(n-1)$

The $3^{\text {rd }}$-order DTTI $\Delta \Sigma$ modulator which is shown in Figure 2 is derived directly from the time domain equation set (6) and (9).

The motive behind sharing integrators is to eliminate the instability that can arise from the DC offset mismatch of the two individual integrator set based two channel interleaving case. The DTTI $\Delta \Sigma$ modulators need an input demultiplexer which samples the input signal at the highest clock frequency of the DTTI $\Delta \Sigma$ modulator and distributes it between channels. This fast demultiplexer is a limiting factor for the performance of the DTTI $\Delta \Sigma$ modulators. This architecture does not need an input demultiplexer and the input signal is shared between channels [3],[5]. Removing the input demultiplexer has no effect on the NTF of the DTTI $\Delta \Sigma$ modulator but it causes some notches in its Signal Transfer Function (STF) at the following frequencies $0.5 F_{c l k}, 1.5 F_{c l k}, 2.5 F_{c l k}, 3.5 F_{c l k}, \ldots$ which is shown in Figure 5 where $F_{c l k}$ is the clock frequency of the DTTI $\Delta \Sigma$ modulator [5].

\section{B. DELAYLESS FEEDBACK PATH PROBLEM IN TI $\Delta \Sigma$ MODULATORS}

This is the issue that forms the focus of this paper which makes implementation of Time Interleaved (TI) $\Delta \Sigma$ modulators with shared integrators impractical and it is called the "delayless feedback path problem" that comes from equation (6.c) in which $v_{31}(n)$ (the input of quantizer Q1) is directly linked to $y_{2}(n)$. This means that the output of the second quantizer $(\mathrm{Q} 2)$ is connected to the input of another quantizer (Q1) without any delay [7]. One method to eliminate the delayless path is to move this feedback to the digital domain instead of performing it in the analog domain [5]. The disadvantage of this method is that the first quantizer (Q1) requires more comparators than the number of the comparators in the second quantizer (Q2) [7]. The second method is to use a sample-and-hold in front of the first quantizer (Q1) and quantizing the signal when the output of DAC2 is ready [3]. This method needs a complicated timing generator, a sampleand-hold and also faster integrators. The third method which is our proposed method is based on substituting $v_{32}(n)$ for $y_{2}(n)$ in equation (6.c). This method increases the quantization noise of the quantizer Q2 at the output $y(n)$. To better understand how this works we shall perform a step by step 


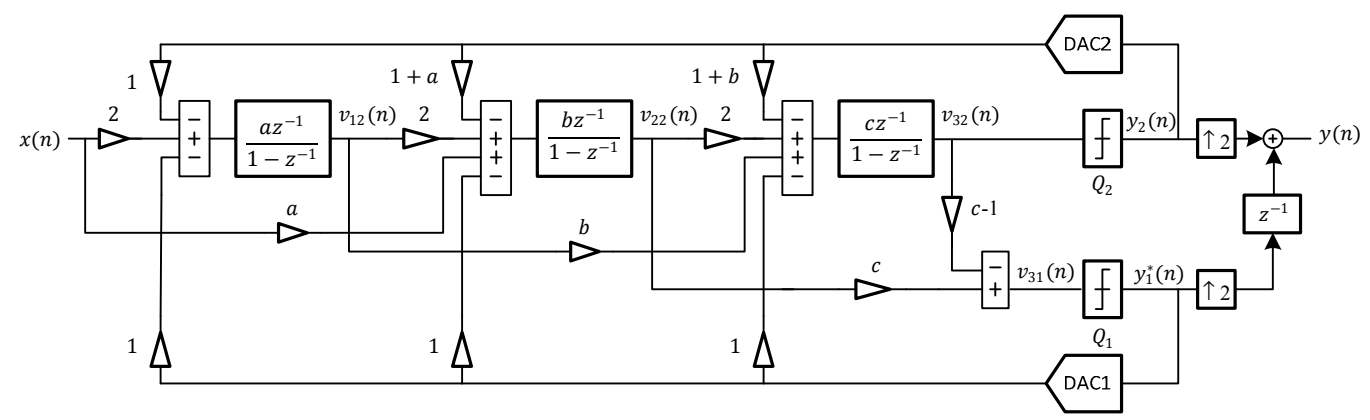

Figure 3: The proposed $3^{\text {rd }}$-order DTTI $\Delta \Sigma$ modulator with shared integrators and without delayless feedback path.

mathematical analysis of what happens. Quantizer Q1 and Q2 quantize the signal $v_{31}(n)$ and $v_{32}(n)$ as follows:

$y_{1}(n)=Q\left[v_{31}(n)\right]=v_{31}(n)+e_{1}(n)$

$y_{2}(n)=Q\left[v_{32}(n)\right]=v_{32}(n)+e_{2}(n)$

Where $e_{1}(n)$ and $e_{2}(n)$ represent the quantization noise of the quantizer Q1 and Q2 respectively. Equation (12) is derived by substituting (6.c) into (10):

$y_{1}(n)=c v_{22}(n)-c y_{2}(n)+v_{32}(n)+e_{1}(n)$

Equation (13) is given by substituting $v_{32}(n)$ for $y_{2}(n)$ into equation (12). The output of Q1 is called $y_{1}^{*}(n)$ in (13):

$y_{1}^{*}(n)=c v_{22}(n)-(c-1) v_{32}(n)+e_{1}(n)$

$y_{1}^{*}(n)$ approximates $y_{1}(n)$ in the DTTI $\Delta \Sigma$ modulator. The error which is induced by this method is presented by equation (14). This error does not cause instability in the modulator but increases quantization noise of quantizer Q2 at the output $y(n)$ followed by degrading the SNR of the modulator.

error $=y_{1}^{*}(n)-y_{1}(n)=c y_{2}(n)-c v_{32}(n)=c e_{2}(n)$

The resulting $3^{\text {rd }}$-order DTTI $\Delta \Sigma$ modulator is shown in Figure 3. The Signal Transfer Function (STF) and Noise Transfer Function (NTF) can be obtained by determining the loop filters of the modulator. In this design, the DTTI $\Delta \Sigma$ modulator has six loop filters $\left(F F_{1}(z), F F_{2}(z), H_{1}(z)\right.$, $H_{2}(z), H_{3}(z)$ and $\left.H_{4}(z)\right)$ and can be determined with the help of the symbolic toolbox of MATLAB. These loop filters for the DTTI $\Delta \Sigma$ modulator are as depicted in Figure 4. The STF and NTFs can be formulated by performing the following algebraic analysis:

$Y(z)=Y_{1}^{*}\left(z^{2}\right) z^{-1}+Y_{2}\left(z^{2}\right)$

$Y(z)=\operatorname{STF}(z) X(z)+N T F_{1}(z) E_{1}(z)+N T F_{2}(z) E_{2}(z)$

$N T F_{1}(z)=\frac{H_{3}\left(z^{2}\right)+\left[1-H_{4}\left(z^{2}\right)\right] z^{-1}}{\left[1-H_{1}\left(z^{2}\right)\right]\left[1-H_{4}\left(z^{2}\right)\right]-H_{2}\left(z^{2}\right) H_{3}\left(z^{2}\right)}$

$N T F_{2}(z)=\frac{\left[1-H_{1}\left(z^{2}\right)\right]+H_{2}\left(z^{2}\right) z^{-1}}{\left[1-H_{1}\left(z^{2}\right)\right]\left[1-H_{4}\left(z^{2}\right)\right]-H_{2}\left(z^{2}\right) H_{3}\left(z^{2}\right)}$

$\operatorname{STF}(z)=N T F_{1}(z) F F_{1}\left(z^{2}\right)+N T F_{2}(z) F F_{2}\left(z^{2}\right)$

Where $\operatorname{STF}(z), N T F_{1}(z)$ and $N T F_{2}(z)$ represent the signal transfer function from $x(n)$ to $y(n)$, the noise transfer function from $e_{1}(n)$ to $y(n)$ and the noise transfer function from $e_{2}(n)$ to $y(n)$ respectively. The $z^{2}$ terms show the effect of the upsamplers in the modulator.

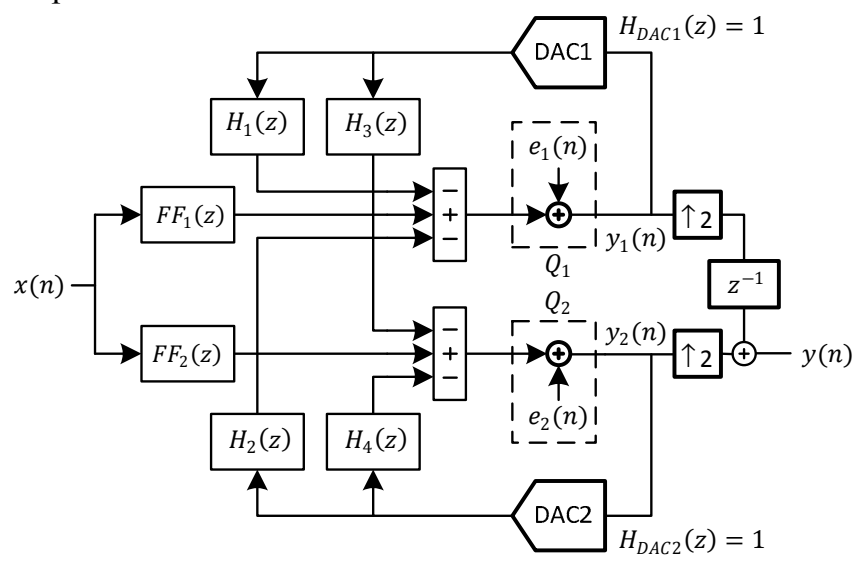

Figure 4: The block diagram of a DTTI $\Delta \Sigma$ modulator.

The conventional $\Delta \Sigma$ modulator is designed through the use of the delta-sigma toolbox from MATLAB to operate at $160 \mathrm{MHz}$ clock frequency and OSR of 16 . The modulator coefficients are $\{a, b, c\}=\{0.333,1,3\}$. The STF and NTFs of the DTTI $\Delta \Sigma$ modulator are given by (20), (21) and (22).

$\operatorname{STF}(z)=z^{-3}\left(1+z^{-1}\right)$

$N T F_{1}(z)=z^{-1}\left(1-z^{-1}\right)^{3}$

$N T F_{2}(z)=\left(1+z^{-1}\right)\left(1-z^{-1}\right)^{3}$

Figure 5 compares the STFs of the DT and the DTTI $\Delta \Sigma$ modulator. STF of DT $\Delta \Sigma$ modulator is flat but STF of DTTI $\Delta \Sigma$ modulator has a notch at half sampling frequency resulting from $\left(1+z^{-1}\right)$ term in (20). Figure 6 shows the NTFs of the DT and the DTTI $\Delta \Sigma$ modulator. NTFs of the DTTI $\Delta \Sigma$ modulator shape the quantization noise more than the NTF of the DT $\Delta \Sigma$ modulator, however, $N T F_{2}(z)$ shapes noise less than $N T F_{1}(z)$ as a result of approximating $y_{1}(n)$ in (13).

\section{SIMULATION RESULTS}

The DT and the DTTI $\Delta \Sigma$ modulator have been simulated using the SIMULINK toolbox of MATLAB and all nonidealities such as finite dc gain, slew-rate and bandwidth of the opamps, the DAC mismatches and offsets of the quantizers have been modelled and their effects on the performance of the modulator have been investigated. The quantizers of the 
DT and the DTTI $\Delta \Sigma$ modulator have 4bits of resolution in this design. The output spectra of the DT and the DTTI $\Delta \Sigma$ modulator for a $2.4462 \mathrm{MHz}$ input and clock frequencies of $160 \mathrm{MHz}$ and $320 \mathrm{MHz}$ respectively are plotted in Figure 7. The output spectra of the DTTI $\Delta \Sigma$ modulator is shaped more than the conventional DT $\Delta \Sigma$ modulator. The SNDRs of the conventional DT and the DTTI $\Delta \Sigma$ modulator are $64.5 \mathrm{~dB}$ and $76.5 \mathrm{~dB}$ respectively. Therefore in this particular case, the SNDRs of the DTTI $\Delta \Sigma$ modulators is improved by $11 \mathrm{~dB}$.

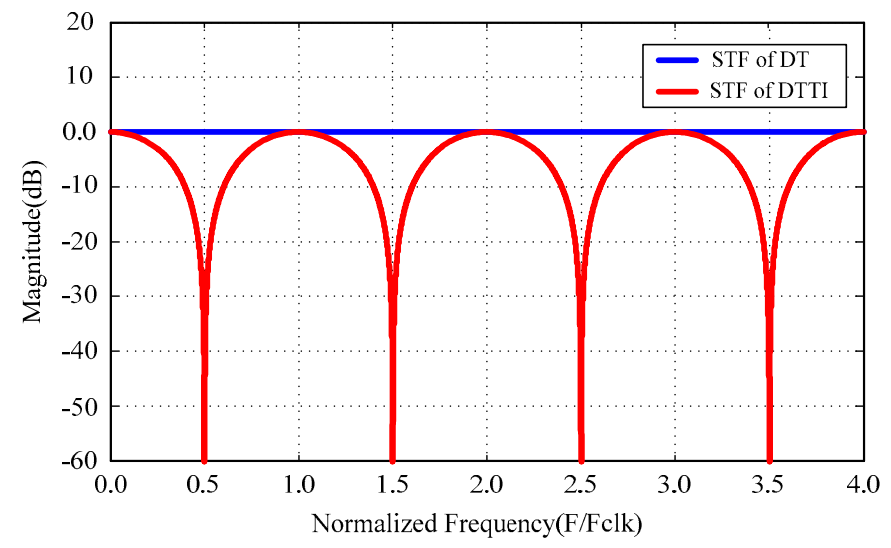

Figure 5: The STFs of the DT and the DTTI $\Delta \Sigma$ modulator.

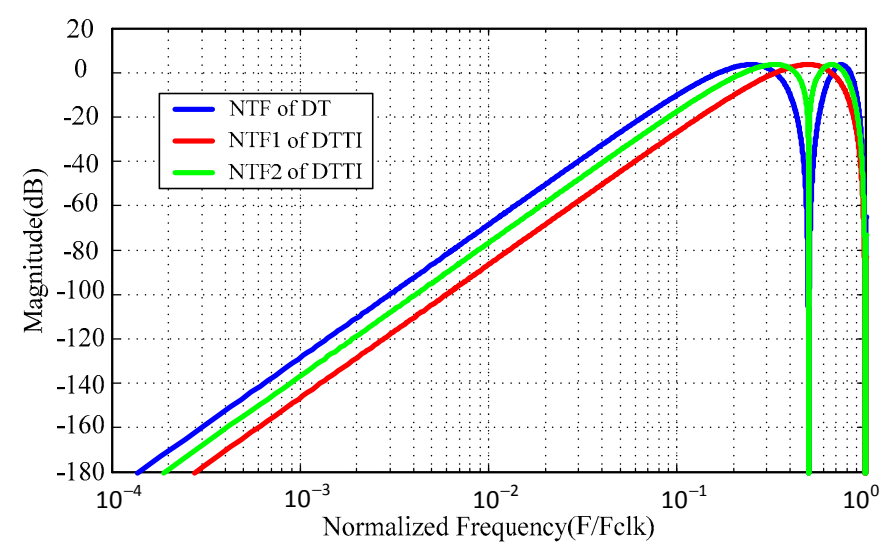

Figure 6: The NTFs of the DT and the DTTI $\Delta \Sigma$ modulator.

\section{CONCLUSION}

In this paper a $3^{\text {rd }}$-order DTTI $\Delta \Sigma$ modulator with shared integrators between two-path and an OSR of 16 has been designed and simulated in the SIMULINK environment of MATLAB to operate at a sampling rate of $320 \mathrm{MHz}$.. All practical non-idealities have been modelled and investigated. To resolve the delayless feedback path issue resulting from sharing integrators between paths, an approximation method has been proposed. The maximum SNDR obtained from the DTTI $\Delta \Sigma$ modulator is $76.5 \mathrm{~dB}$ which shows an $11 \mathrm{~dB}$ improvement in comparison with the standard single-loop single-path DT $\Delta \Sigma$ modulator.

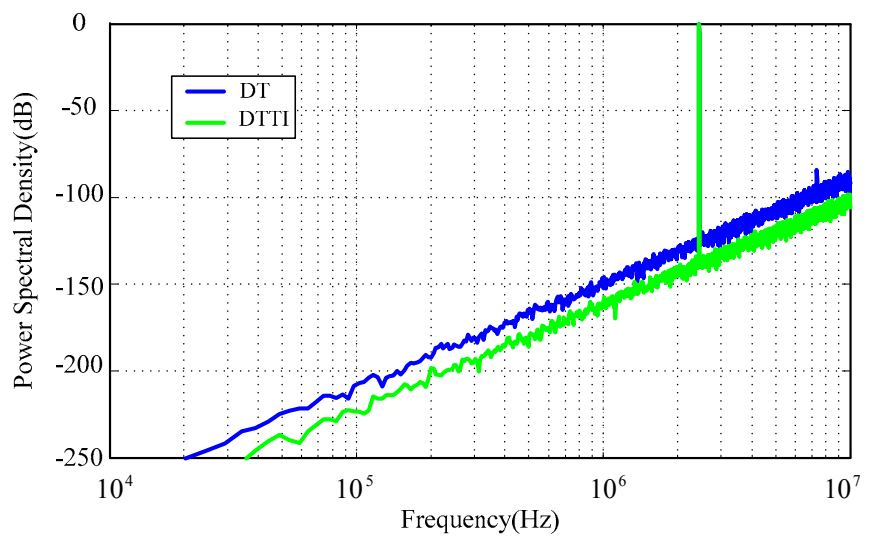

Figure 7: The output spectra of the DT and the DTTI $\Delta \Sigma$ modulator for a $2.4462 \mathrm{MHz}$ input with clock frequencies of $160 \mathrm{MHz}$ and $320 \mathrm{MHz}$ respectively.

\section{REFERENCES}

[1] M. Ortmanns and F. Gerfers, Continuous-Time Sigma-Delta A/D Conversion. Berlin: Springer, 2006.

[2] D. Yang, F. F. Dai, W. N. S. Yin and R. C. Jaeger," Delta-Sigma Modulation for Direct Digital Frequency Synthesis," IEEE Trans. Very Large Scale Integr.(VLSI) Syst., vol. 17, no. 6, pp. 793-802, June 2009.

[3] T. C. Caldwell and D. A. Johns," A Time-Interleaved Continuous-Time $\Delta \Sigma$ Modulator with 20-MHz Signal Bandwidth," IEEE J. Solid-State Circuits, vol. 41, no. 7, pp. 1578-1588, July 2006.

[4] R. Khoini-Poorfard, L. B. Lim and D. A. Johns," Time-Interleaved Oversampling A/D Converters: Theory and Practice," IEEE Trans. Circuits Syst. II, vol. 44, no. 8, pp. 634-645, Aug. 1997.

[5] A. Gharbiya, T. C. Caldwell and D. A. Johns," High-Speed Oversampling Analog-To-Digital Converters," International Journal of High Speed Electronics and Systems, pp. 1-21, 2005.

[6] M. Kozak and I. Kale, "Novel Topologies for Time-Interleaved DeltaSigma Modulators," IEEE Trans. Circuits Syst. II, Analog Digit. Signal Process, vol. 47, no. 7, pp. 639-654, Jul. 2000.

[7] Y. Zhang and A. Leuciuc," A 1.8V Continuous-Time Delta-Sigma Modulator with $2.5 \mathrm{MHz}$ Bandwidth," IEEE MWSCAS, pp. 140-143, 2002.

[8] K. S. Lee, Y. Choi and F. Maloberti," Domino Free 4-Path TimeInterleaved Second Order Sigma-Delta Modulator," IEEE ISCAS, pp. 473-476, 2004.

[9] A. Gharbiya and D. A. Johns," Combining Multipath and Single-Path Time-Interleaved Delta-Sigma Modulators," IEEE Trans. Circuits Syst. II, vol. 55, no. 12, pp. 1224-1228, Dec. 2008.

[10] B. Xia, S. Yan and E. S. Sinencio,"An RC Time Constant Auto-Tuning Structure For High Linearity Continuous-Time Delta-Sigma Modulators and Active Filters," IEEE Trans. Circuits Syst. I, vol. 54, no. 11, pp. 2179-2188, Nov. 2004.

[11] K. Lee, S. Kwon and F. Maloberti,"A Power-Efficient Two-Channel Time-Interleaved $\Delta \Sigma$ Modulator for Broadband Applications," IEEE J. Solid-State Circuits, vol.42, no.6, pp. 1206-1215, June 2007.

[12] K. Lee and F. Maloberti," Time-Interleaved Sigma-Delta Modulator Using Output Prediction Scheme" IEEE Trans. Circuits Syst. II, vol. 51, no. 10 , pp. 537-541, Oct. 2004.

[13] M. Kozak, M. Karaman, and I. Kale, "Efficient Architectures for TimeInterleaved Oversampling Delta-Sigma Converters," IEEE Trans. Circuits Syst. II, Analog Digit. Signal Process, vol. 47, no. 8, pp. 802810, Aug. 2000.

[14] A. Gharbiya and D. A. Johns," On The Implementation of Input Feedforward Delta-Sigma Modulator," IEEE Trans. Circuits Syst. II, vol. 53, no. 6, pp. 453-457, June. 2006.

[15] T. C. Caldwell," Time-Interleaved Continuous-Time Delta-Sigma Modulators," MASc Thesis, University of Toronto, Toronto, Ontario, Canada, 2004. 